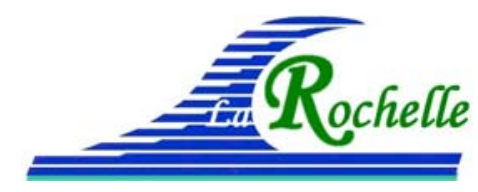

XVèmes Journées Nationales Génie Côtier - Génie Civil

La Rochelle, 29 au 31 mai 2018

DOI:10.5150/jngcgc.2018.025 C Editions Paralia CFL

disponible en ligne - http://www.paralia.fr - available online

\title{
Evolution contemporaine du littoral Ouest-Méditerranée depuis les années 2000
}

\author{
Mathieu GERVAIS ${ }^{1}$, Loïc INIZAN ${ }^{1}$, \\ Hugues HEURTEFEUX ${ }^{1}$, Delphine BOULET ${ }^{1}$
}

\begin{abstract}
1. EID-Méditerranée, 165 Rue Paul Rimbaud, 34000 Montpellier, France. mgervais@eid-med.org ; linizan@eid-med.org ; hheurtefeux@eid-med.org ; dboulet@eid-med.org
\end{abstract}

\section{Résumé :}

Le littoral sableux du Golfe du Lion montre de nombreux secteurs à enjeux, où les infrastructures construites trop proches du trait de côte sont aujourd'hui exposées de manière trop fréquente à l'érosion et à la submersion marine lors des tempêtes.

Des questions demeurent encore sur la dynamique à court terme des plages, et des paramètres qui gouvernent les taux de dépôt/érosion d'une année sur l'autre sur chacun des secteurs de ce littoral présentant une forte continuité sédimentaire. Nous manquons en cela de recul et d'une vision large échelle sur la contribution relative des processus de dérive, de pertes au large et d'apports par les 6 principaux petits fleuves côtiers de la région. Les crues se montrent en effet susceptibles de recharger, sur quelques kilomètres de linéaire côtier, les avant-côtes en sable.

Notre étude investit un jeu de données unique de profils topo-bathymétriques acquis par l'EID-Méditerranée depuis les années 2000, avec plus de 1015 transects bathymétriques et 1646 transects topographiques levés au DGPS et au sondeur. Ces données seront complétées par des levés réalisés par d'autres organismes dans le cadre de projet de recherche ou de suivis de travaux. L'analyse est actuellement en cours à l'EIDMéditerranée, mais les premiers résultats sur les volumes de sables perdus ou gagnés sur l'avant-côte, en particulier sur le système de barres immergés sont présentés sur une cellule hydro-sédimentaire de $12 \mathrm{~km}$ environ entre l'Orb et l'Hérault.

Les résultats montrent clairement des transferts longitudinaux de sable saisonniers à l'échelle de la cellule hydro-sédimentaire limitée par des grandes digues, mais aussi l'effet "bénéfique ou néfaste" de certains hivers, en lien avec la chronique des tempêtes. L'effet des houles est nettement modulé par l'importance des crues s'étant produites au même moment au cours de la saison humide d'octobre à mai.

Mots-clés :

Erosion, Profils topo, Bathymétrie, Dérive Sédimentaire littorale, Tempête, Crue, Rechargement, Avant-côte. 


\section{Thème 2 - Dynamique sédimentaire}

\section{Introduction}

Le littoral sableux ouest-méditerranéen, si l'on exclut le delta de Camargue, est composé essentiellement de minces cordons littoraux représentant des volumes de sables limités. Depuis les années 2000, la recherche et le nombre d'études sur la dynamique sableuse de la région est en plein essor. En ce qui concerne l'estimation des vitesses d'érosion des plages, la majorité des études utilise la détection du trait de côte (milieu de la zone de jet de rive en temps calme) à partir d'images aériennes (ex. : SABATIER \& HANOT, 2012). Les travaux de recherche morphodynamiques se restreignent souvent à des secteurs trop limités du littoral, et le lien entre changement morphologique (ex. : mouvement des barres) et transport large-échelle du sable n'est pas bien dissocié. Seule l'évolution séculaire des volumes de sables disponibles de toute l'avant-côte régionale a été décrite par l'atlas de BRUNEL et al. (2012). L'atlas identifie les zones du Golfe du Lion où l'érosion des fonds se révèle critique, et où celle-ci s'accélère. Dans le même temps, la connaissance des phénomènes de transport liés à la dérive littorale s'est nettement améliorée grâce au progrès des modèles de prévision des vagues et des ré-analyses climatiques (KULLING, 2017).

Si aujourd'hui les secteurs en érosion sont bien connus, de même que les ordres de grandeur de la dérive, les causes de la variabilité interannuelle des pertes et des gains de sable sur un site ne sont que partiellement expliquées.

Par exemple, on n'observe pas le même volume d'un été à l'autre, et en particulier sur l'avant-côte. Les travaux récents de l'OBSCAT dans les Pyrénées-Orientales (LOTHE et al., 2016) montrent que plusieurs systèmes de plage et avant-côte voisins peuvent simultanément voir un retour significatif du sable sur plus de $10 \mathrm{~km}$ de linéaire grâce aux mouvements de sable entre 0 et $-10 \mathrm{~m}$, sur les systèmes de barres où se produit la majorité des changements sableux avec des variations 10 fois supérieures à celles de la plage aérienne. Nous pensons que l'apport des fleuves a souvent été négligé, que les épisodes violents de crue se révèleraient encore aujourd'hui essentiels au fonctionnement du système sableux littoral.

\section{Méthodologie}

\subsection{Projet}

L'objectif premier du projet est d'actualiser la connaissance sur l'érosion des volumes de plages émergées, mais aussi immergées, sur la période récente 2000-2017, à partir d'une base de données très fournie de profils topo-bathymétriques encore jamais utilisée dans son intégralité. Cette base comprend 1015 profils bathymétriques et 1646 profils topographiques concernant essentiellement le département de l'Hérault. Un test a montré que quelques profils par kilométrique permettent de rendre compte convenablement des variations de volumes. L'objectif scientifique est de réfléchir aux 


\section{XVèmes Journées Nationales Génie Côtier - Génie Civil \\ La Rochelle, 29 au 31 mai 2018}

problématiques décrites précédemment, notamment le rôle des tempêtes, de la dérive, et des apports sableux par les petits fleuves côtiers aux régimes torrentiels.

\subsection{Site d'étude}

Au stade actuel du projet, seule l'évolution des 49 profils situés entre les fleuves Orb et Hérault, entre Valras et le grau d'Agde (cf. figure 1) a été analysée. Nous présentons ici la dynamique de la zone immergée entre le trait de côte et une profondeur standard de -7 $\mathrm{m}$, correspondant à la profondeur de recouvrement des profils dans le temps. La marge d'erreur sur l'estimation de la variation du volume sur le profil immergé (environ $700 \mathrm{~m}$ depuis le trait de côte) est de + ou $-30 \mathrm{~m}^{3}$ par mètre linéaire de côte.

Sur ce secteur, la dérive dominante se fait vers le sud-ouest et les plages situées les plus à l'est sont celles qui montrent le plus d'érosion historiquement.

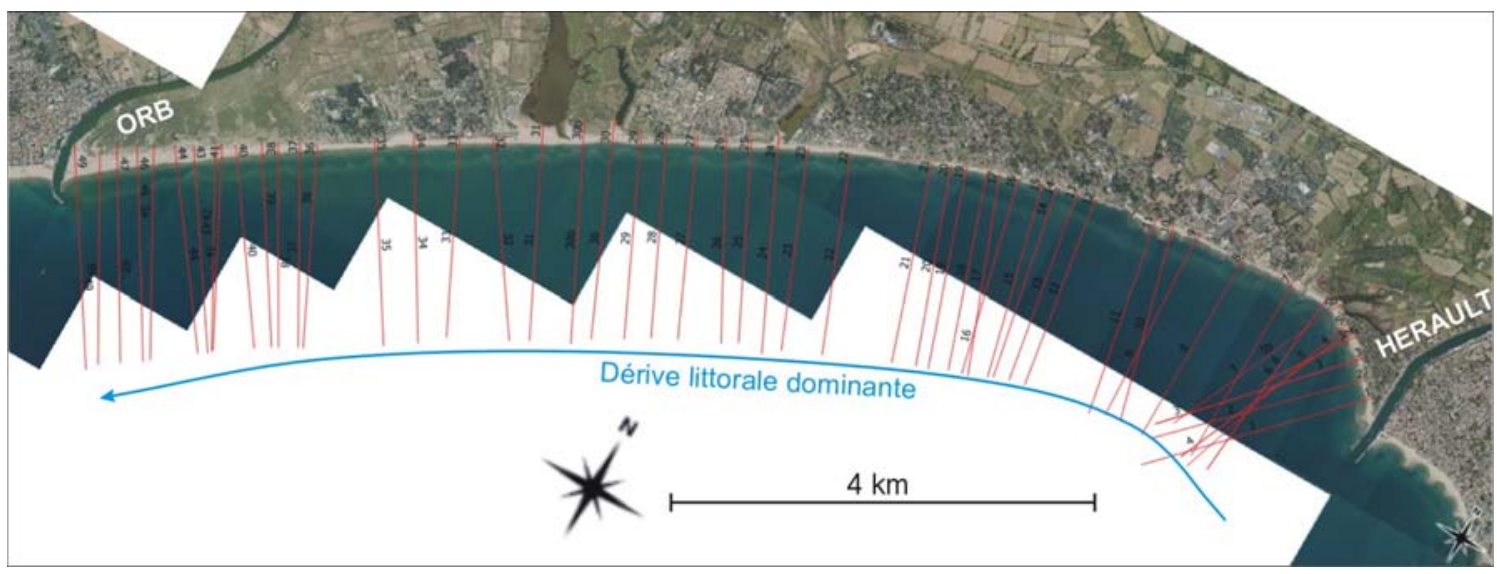

Figure 1. Site d'étude de l'ouest-Hérault (entre Agde et Valras).

\section{Résultats}

\subsection{Conditions de houle et épisodes de crue durant le suivi sur l'ouest Hérault}

Evolution de l'énergie de la houle hivers après hivers

Une analyse a été réalisée sur l'énergie de houle depuis l'hiver 2003-2004 au niveau de la bouée houlographe de Sète. Une estimation satisfaisante de la dérive sur la zone d'étude est donnée en cumulant la puissance longitudinale de la houle sur tout un hiver d'après l'orientation générale du trait de côte sur le secteur $\left(60^{\circ} \mathrm{N}\right.$; comparaison faite avec les modélisation de KULLING, 2017). Cette dérive dépend de la répétition et du nombre de tempêtes par hiver, mais aussi de la présence de tempêtes venant du secteur sud et favorisant une dérive inverse sur la zone d'étude. La figure 2 montre que la dérive a été forte en 2003-2004, 2005-2006, 2010-2011 et 2016-2017. A l'inverse elle a été parfois faible, voir quaisment nulle en 2013-2014. 


\section{Thème 2 - Dynamique sédimentaire}

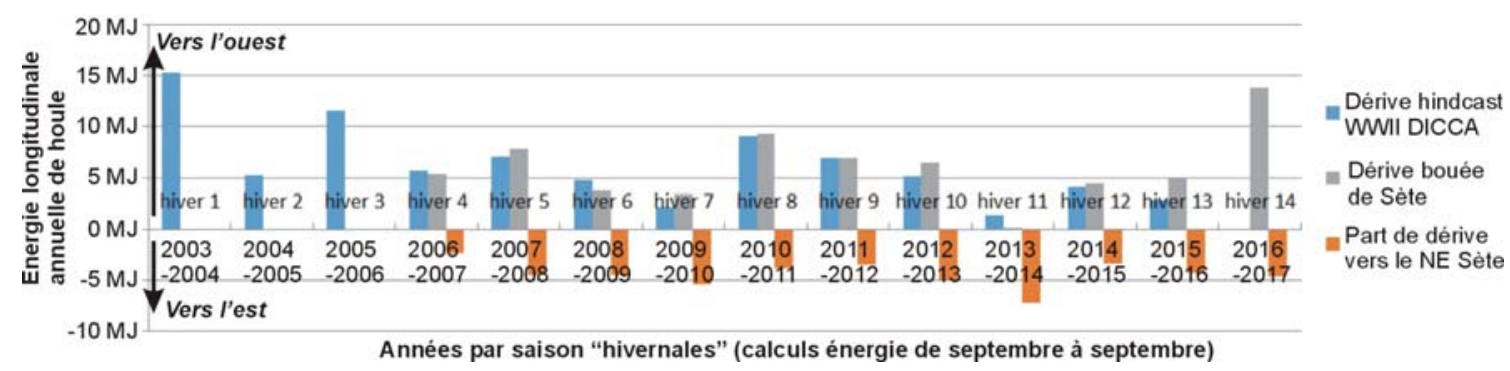

Figure 2. Energie de houle longitudinale cumulée par saison hivernale depuis 20022003 (résultante) donnant une approximation de la vitesse de dérive vers l'ouest sur le site d'étude (en bleu et gris). Une approximation de la dérive inverse vers le NE est donnée en orange. Le modèle de houle du DICCA complète les données anciennes.

Episodes de crues significatifs

La série temporelle du débit de l'Hérault à Agde est présentée en figure 3 suivante.

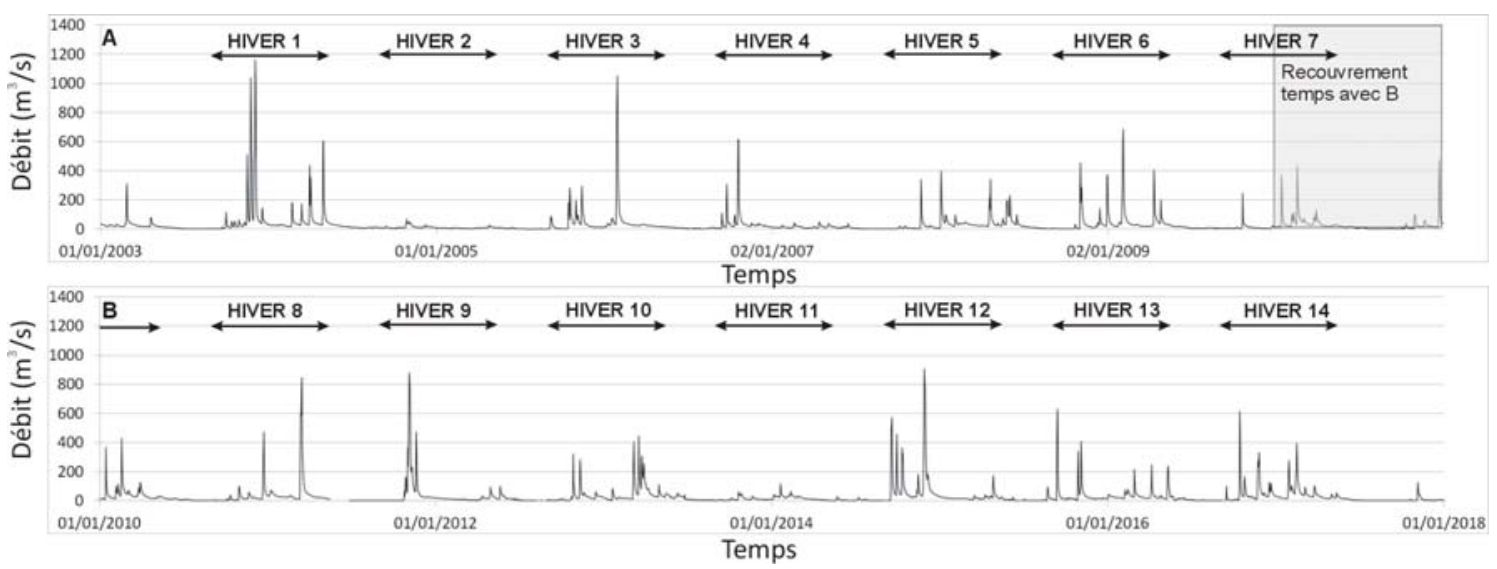

Figure 3. Série temporelle du débit moyen journalier de l'Hérault à Agde de janvier 2003 à janvier 2018 (débit exprimé en $\mathrm{m}^{3} / \mathrm{s}$ ).

Les données montrent certains hivers très secs comme l'hiver 2004-2005 (hiver 2) ou l'hiver 2013-2014 (hiver 11), et à l'inverse certains hivers avec des épisodes de crues très rapides et violentes (crues éclair), comme les crues successives du 25/11/2003 et du 04/12/2003 (hiver 1).

\subsection{Résultat préliminaires sur le secteur ouest-Hérault (de l'Orb à l'Hérault)}

Le Tableau 1 suivant présente la matrice de dépôt et d'érosion pour tous les profils bathymétriques du site entre les principales dates de levé. Les dates de levés masquées par une couleur noire tiennent compte de plusieurs hivers ce qui fausserait l'interprétation de l'évolution interannuelle des dépôts :

- Une érosion majoritaire et très prononcée sur l'ensemble du site d'étude au cours du premier hiver (2003-2004). L'érosion forte sur la partie ouest du site exclut 


\section{XVèmes Journées Nationales Génie Côtier - Génie Civil \\ La Rochelle, 29 au 31 mai 2018}

l'hypothèse d'un transfert simple par la dérive littorale lors des tempêtes les plus extrêmes. Celles-ci semblent provoquer un transfert du sable au-delà de la profondeur de fermeture, ou longitudinalement au-delà de l'Orb. Les crues fortes de l'Hérault n'ont pas suffi à contrebalancer l'érosion sur la partie est du site.

- Un deuxième hiver apparemment peu morphogène puisqu'il n'a pas modifié de manière importante les volumes sur les quelques profils suivis sur la partie centraleouest du site. Cette réponse s'explique probablement par l'absence de crue et la relative "faiblesse" des tempêtes face à l'hiver précédent responsable d'une dérive plus faible et plus équilibrée (autant d'apport que de départ de sable).

- L'hiver 3 (2005-2006), riche en tempêtes et en crues a renforcé l'érosion de l'hiver 2003-2004, mais uniquement sur une petite partie est du site proche de l'Hérault. Le dépôt est majoritaire sur tout le reste du site. On suppose que le dépôt sur toute la zone centrale s'explique par des apports en sable depuis l'Hérault, poussés par la dérive, mais qui n'ont pu suffire à empêcher l'érosion des derniers épisodes de houle "secs", c'est-à-dire non associé à des crues sur la partie est du site.

- L'hiver 4 (2006-2007) produit un dépôt important sur les extrémités est et ouest du site. Après une fin d'hiver sans crues, ce dépôt est certainement la marque d'un retour du sable depuis le large. Aussi, il est possible que l'accumulation aux extrémités de la cellule soit l'effet de la dérive alternant selon deux directions et poussant le sable aux deux extrémités de ce linéaire côtier concave (forme de baie).

- De la même manière, l'hiver 5 (2007-2008) a favorisé le retour du sable par rapport à la situation érosive post-hiver 3 . Il fut donc bénéfique en dépit là encore de quelques épisodes de tempêtes avec des Hs de vagues importantes $(>4 \mathrm{~m})$. Pour ces deux hivers 4 et 5 , il n'est pas à exclure un apport en sable lié aux épisodes de crues modérés mais non pas absents.

- L'hiver 6 (2008-2009) crée de l'érosion puis du dépôt au printemps sur la partie centrale du site. L'apport observé à l'est, proche de l'embouchure est la résultante des 3 hivers précédents, et probablement des apports successifs à l'embouchure.

- L'hiver 7 (2009-2010) alimente la partie est du site, certainement du fait des crues et des épisodes de tempêtes avec des houles venant du sud cet hiver-là (équilibrant et réduisant le bilan de la dérive vers l'est en figure 2).

- L'hiver 8 n'a pu être dissocié de la fin de l'hiver 7 (et de l'été). Il semble provoquer une faible érosion sur la partie est tandis que la partie centrale évolue peu. Dans ce cas, on considère que les nombreuses tempêtes venant de l'est n'ont pas été totalement réduit l'effet de la très forte crue de la fin de l'hiver.

- L'hiver 9 (2011-2012) montre une érosion à l'est et un dépôt sur la partie centrale (mais seuls deux profils donnent cette information). Cette fois apparemment, les crues fortes n'ont pas été suffisantes pour éviter l'érosion à l'est. 
Thème 2 - Dynamique sédimentaire

Tableau 1. Matrice de dépôt et d'érosion $(\mathrm{m} 3 / \mathrm{ml})$ entre chaque date de levé par profil (de l'ouest vers l'est ; les cases en noirs tiennent compte de plusieurs hivers successifs).
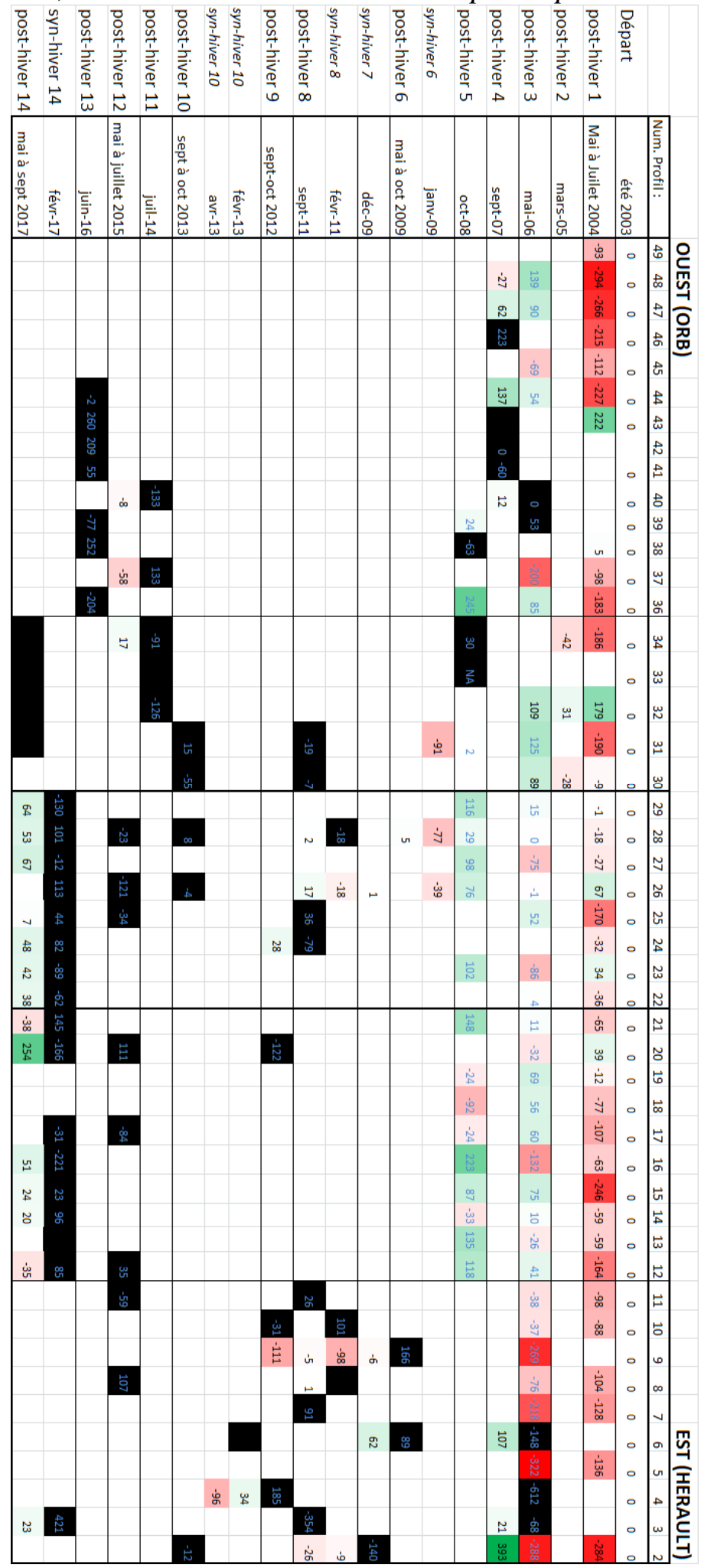


\section{$X V^{\text {èmes }}$ Journées Nationales Génie Côtier - Génie Civil \\ La Rochelle, 29 au 31 mai 2018}

- L'hiver 10 (2012- 2013) voit successivement des phases de dépôt et d'érosion sur la partie est du site, s'expliquant potentiellement par des tempêtes plus ou moins "compensées" par des crues concomitantes : par exemple les tempêtes avant février 2013 sont contrebalancées par des apports, excepté la forte tempête du 05 mars 2013.

- Suite à l'hiver 11 (2013-2014), aucun profil n'a été levé, ce qui ne permet pas de faire une interprétation sur la dynamique de transport.

- L'hiver 12 (2014-2015) produit une érosion majoritaire sur les profils de la partie ouest du site, certainement à cause des dernières tempêtes de la saison venant du sud.

- L'hiver 13 (2015-2016) semble favorable au retour du sable, mais où aucun profil n'a pu être suivi de manière répétée par rapport aux saisons précédentes.

- Un hiver 14 (2016-2017) indiscutablement favorable au retour du sable sur la totalité du linéaire côtier, malgré un début d'hiver qui semblait érosif. Notons que dans ce cas les tempêtes de l'hiver ont à plusieurs reprises été associées à des débits importants à l'embouchure du fleuve Hérault.

Les interprétations qui sont proposées quant aux causes de l'érosion ou du dépôt sur les profils au terme de chacune des 14 périodes hivernales révèlent indiscutablement que le transport longitudinal parait la cause première des variations longitudinales de la sédimentation: des portions du site sont plus érodées que d'autres certaines années, indépendamment de la proximité du fleuve (cf. érosion plus forte à l'ouest l'hiver 1 et plus forte à l'est l'hiver 3 en Tableau 1). Comme il est connu sur la zone, les épisodes érosifs sont généralement bien plus forts sur la partie est du site qui est un hotspot d'érosion par effet de la dérive vers l'ouest-sud-ouest nettement plus représentée lors des fortes houles. Néanmoins, il est montré ici que les dépôts peuvent être aussi parfois plus forts proche de l'embouchure de l'Hérault (et non, comme habituellement, contre l'embouchure de l'Orb). Dans ce cas il est difficile de savoir si cela est le fait d'épisodes répétés de houles venant du secteur sud, ordinairement plus rares, ou la conséquence des apports par le fleuve Hérault lors des crues (épisodes cévenols).

Au final, même si l'effet bénéfique des crues n'a pu être dissocié de manière évidente, il est inévitablement un contributeur essentiel au stock sédimentaire et à la stabilité des plages dans le temps, en particulier sur cette zone où plusieurs dizaines de milliers de mètres-cubes sont perdus vers l'ouest chaque année. Il est assumé que le déficit en sable lié à l'interruption importante de la dérive par la digue de l'Hérault est amoindri par les apports des crues. Ces crues rechargent les bancs de sable de l'embouchure qui servent de future zone source.

L'ordre de grandeur des changements de volumes saisonniers sur l'avant-côte exclut que les dépôts se soient fait sur la plage émergée uniquement, ce qui sera vérifié prochainement par l'analyse des profils topographiques. Seul un traçage du sable ou de la modélisation morphodynamique large échelle, incluant les apports solides de l'Hérault permettrait d'expliquer toute la complexité des phénomènes observés. De même, ce travail fait l'hypothèse que les pertes au large lors des tempêtes et les retours 


\section{Thème 2 - Dynamique sédimentaire}

du sable sont marginaux, du moins d'un ordre de grandeur inférieur à la répartition des volumes sableux par la dérive et les rechargements par le fleuve qui s'opèrent annuellement. Notre jeu de données montre que lors de certains hivers, comme l'hiver 1 , tout le site d'étude devient déficitaire en sable. Nous pensons que dans ce cas, le sable franchit la digue de l'Orb à l'ouest et que la cellule étudiée n'est plus un système fermé. Lors des tempêtes, du sable est inévitablement propulsé vers le large contre la digue de l'Orb par des courants d'arrachement, puis repris par les vagues pour alimenter les cellules hydro-sédimentaires plus au sud-ouest.

\section{Conclusion}

Ce travail aborde pour la première fois dans la région ouest-méditerranéenne une vision longitudinale des processus de dépôt et d'érosion sur la partie immergée de l'avant-côte à l'échelle interannuelle. Un jeu de données unique de 14 années de profils encadrants des saisons hivernales permet d'interpréter l'effet de la dérive et des accumulations sur les grandes digues qui bornent les cellules hydro-sédimentaires littorales de la région (ports ou embouchures) et permet d'étudier les déplacements large-échelle du sable. En première analyse, certaines années se montrent érosives pour la totalité du cordon malgré de forts apports par le fleuve voisin, à cause indéniablement d'épisodes de tempêtes extrêmes. Pourtant, d'autres années plus calmes mais néanmoins riches en tempêtes semblent favorables au dépôt. Nous expliquons cela par une compétition des facteurs marins/fluviaux, même si des investigations par modélisation doivent être menées. Le dépôt d'ampleur variable longitudinalement s'explique soit pas l'action de la dérive littorale, pouvant être bidirectionnel et d'importance variable selon les changements d'orientation du trait de côte, soit par l'apport des fleuves en sables repris par les vagues depuis l'embouchure.

\section{Références bibliographiques}

BRUNEL C., CERTAIN R., ROBIN N., ALEMAN N., RAYNAL O., BARUSSEAU J.P., SABATIER F. (2012). Atlas de l'évolution des fonds et des budgets sédimentaires séculaires de l'avant côte du Languedoc-Roussillon 1895 / 1984 / 2009. CEFREM 2012.

KULLING B. (2017). Déformation du rivage et dérive littorale des plages du Golfe du Lion. Thèse de doctorat de l'université d'Aix-Marseille.

LOTHE M., BALOUIN Y., PALVADEAU E., STEPANIAN A. BELON R., Coll. MALDAN F., BODÉRÉ G., LATAPY A., BOUVIER C., SIBERT V., DAILLOUX D., SABARICH L. (2016). Observatoire de la côte sableuse catalane - OBSCAT: rapport technique d'année 3. Rapport final. BRGM/RP- 66077-FR, 226 p., 208 ill., 2 ann.

SABATIER F., HANOT B. (2012). Les variations du rivage du Languedoc-Roussillon. Rapport de phase 1. Rapport pour la DREAL-LR, Université Aix Marseille I, laboratoire CEREGE UMR 7073, 47 p. (+annexes) 